\title{
EQUIVALENCE OF A PROBLEM IN MEASURE THEORY TO A PROBLEM IN THE THEORY OF VECTOR LATTICES
}

\author{
GEORGE W. MACKEY
}

In connection with some other work of the author a question arises concerning the form of the general bounded linear ${ }^{1}$ functional on certain vector lattices. ${ }^{2}$ It is the purpose of this note to show that this question is completely equivalent to a question in measure theory which has been discussed and partially answered by Ulam [2].

Let $S$ be an abstract set and let $\mathfrak{F}$ be the vector lattice of all realvalued functions defined on $S$. For each $s_{0}$ in $S$ the function $F$ on $\mathfrak{F}$ such that $F(f)=f\left(s_{0}\right)$ for all $f$ in $\mathfrak{F}$ is clearly a linear functional. We shall call it the point functional belonging to $s_{0}$ or simply a point functional. Obviously every point functional and hence every finite linear combination of point functionals is bounded in the sense that it carries every bounded subset of $\mathfrak{F}$ into a bounded set of real numbers. Our question is as to whether every bounded linear functional on $\mathfrak{F}$ is a finite linear combination of point functionals. We shall show that this is the case if and only if there exists no countably additive measure $\alpha$ which is defined for all subsets of $S$, which is zero at points, which takes on only the values zero and one, and which does not vanish identically.

It is well known that every bounded linear functional on a vector lattice is a difference of non-negative linear functionals ${ }^{3}$ and it is obvious that a non-negative linear functional is bounded. It follows that we need only consider non-negative linear functionals. Passages from a measure to a non-negative linear functional defined on a class of functions and vice versa are of frequent occurrence in mathematical literature. The proof of our theorem rests basically on the fact that when the methods used in effecting these passages are applied to the case at hand one obtains a natural one-to-one correspondence between the non-negative linear functionals on $\mathfrak{F}$ and the countably ad-

Received by the editors March 7, 1944.

1 By a linear functional we mean a functional which is additive and homogeneous; that is, one which preserves linear combinations.

${ }^{2}$ See chap. 7 of $[1]$ for definitions of the terms from the theory of vector lattices which we shall use. Numbers in brackets refer to the references cited at the end of the paper.

${ }^{3}$ This is proved on p. 115 of [1] for additive functionals and it is clear that an additive non-negative functional must be linear. 
ditive measures which are defined on all subsets of $S$ and take on only a finite number of different values.

LEMMA 1. If $P$ is a non-negative linear functional on $\mathfrak{F}$ and if $\phi_{1}, \phi_{2}, \cdots$ are the characteristic functions of mutually disjoint subsets of $S$ then at most a finite number of the numbers $P\left(\phi_{1}\right), P\left(\phi_{2}\right), \ldots$ are different from zero.

PROOF. Suppose the contrary and change the notation so that $P\left(\phi_{1}\right), P\left(\phi_{2}\right), \cdots$ are all different from zero. Let $E_{i}$ be the set whose characteristic function is $\phi_{i}, i=1,2, \ldots$, and let $E=E_{1} \cup E_{2} \cup \ldots$. For each $s$ in $E_{i}$ let $g(s)=1 / P\left(\phi_{i}\right)$ and for each $s$ in $S-E$ let $g(s)=0$. Then for each $n=1,2, \cdots, g \geqq\left(\phi_{1} / P\left(\phi_{1}\right)\right)+\left(\phi_{2} / P\left(\phi_{2}\right)\right)+\cdots$ $+\left(\phi_{n} / P\left(\phi_{n}\right)\right)$. Hence $P(g) \geqq 1+1+\cdots+1=n$ for $n=1,2, \cdots$ and this is impossible.

LEMMa 2. If $P$ is a non-negative linear functional on $₹$ then $P=P_{1}+P_{2}$ where $P_{1}$ is a finite linear combination of point functionals and $P_{2}$ is a non-negative linear functional which vanishes on characteristic functions of points.

PRoof. By Lemma 1 there are at most a finite number of points in $S$ whose characteristic functions are not taken into zero by $P$. Let $s_{1}, s_{2}, \cdots, s_{r}$ include all of these points and let $P_{1}(f)=P\left(\phi_{1}\right) f\left(s_{1}\right)$ $+P\left(\phi_{2}\right) f\left(s_{2}\right)+\cdots+P\left(\phi_{r}\right) f\left(s_{r}\right)$ for all $f$ in $\mathfrak{F}$ where $\phi_{i}$ is the characteristic function of $s_{i}$ for $i=1,2, \cdots, r$. Then let $P_{2}=P-P_{1}$. It is obvious that $P_{2}$ is linear and vanishes on characteristic functions of points. Finally since any non-negative member of $\mathfrak{F}$ is the sum of a function which vanishes at the $s_{i}$ and a linear combination of the $\phi_{i}$ with non-negative coefficients it is easily verified that $P_{2}$ is nonnegative.

LEMMA 3. If $P$ is a non-negative linear functional on $\mathfrak{F}$ which vanishes on all characteristic functions then $P$ vanishes identically.

PRoof. It is obviously sufficient to show that $P(f)=0$ whenever $f \geqq 0$. For each $i=1,2, \cdots$ let $E_{i}$ be the set of all $s$ in $S$ such that $i-1 \leqq f(s)<i$ and let $\phi_{i}$ be the characteristic function of $E_{i}$. For each $s$ in $S$ there exists one and only one $i=1,2, \cdots$ such that $s \in E_{i}$. Let $g(s)=i f(s)$. For each $n=1,2, \cdots, g-\phi_{1} g-\phi_{2} g-\cdots-\phi_{n-1} g$ $\geqq n\left(f-\phi_{1} f-\phi_{2} f-\cdots-\phi_{n-1} f\right)$ and it is obvious that $P(h)=0$ whenever $h$ is a bounded function. Hence for each $n=1,2, \cdots, 0 \leqq P(f)$ $\leqq P(g) / n$. Thus $P(f)=0$.

LEMMA 4. Let $\phi_{1}, \phi_{2}, \cdots$ be the characteristic functions of mutually 
disjoint subsets of $S$ and let $\phi$ be the characteristic function of their union. Then if $P$ is a non-negative linear functional on $F, P(\phi)=P\left(\phi_{1}\right)$ $+P\left(\phi_{2}\right)+\cdots$.

Proof. By Lemma 1 there exists $n_{0}$ such that for $n>n_{0}, P\left(\phi_{n}\right)=0$. Since $P\left(\phi-\phi_{1}-\cdots-\phi_{n_{0}}\right)=P(\phi)-P\left(\phi_{1}\right)-\cdots-P\left(\phi_{n_{0}}\right)$ we have only to show that $P\left(\phi-\phi_{1}-\cdots-\phi_{n_{0}}\right)=0$. In other words we may confine ourselves to the case in which $P\left(\phi_{n}\right)=0$ for $n=1,2, \cdots$. For each $s$ in $S$ if $\phi_{n}(s)=1$ for some $n=1,2, \cdots$ let $g(s)=n$; otherwise let $g(s)=0$. Then for each $n=1,2, \cdots, g-\phi_{1}-2 \phi_{2}-\cdots$ $-(n-1) \phi_{n-1} \geqq n\left(\phi-\phi_{1}-\cdots-\phi_{n-1}\right)$. Hence $P(g) \geqq n P(\phi)$. In other words $0 \leqq P(\phi) \leqq P(g) / n$ for $n=1,2, \cdots$ Thus $P(\phi)=0$.

We may now prove our equivalence theorem.

TheOREM. Every bounded linear functional on $\mathfrak{F}$ is a finite linear combination of point functionals if and only if there exists no countably additive measure $\alpha$ defined on all subsets of $S$ which is zero at points, which takes on only the values zero and one and which does not vanish identically.

Proof. Suppose that a measure of the sort described does exist. Let $f$ be an arbitrary member of $\mathfrak{F}$. Using the fact that $\alpha$ takes on only one nonzero value, it is easy to prove the existence of a sequence $I_{1}, I_{2}, \cdots$ of closed intervals on the real line such that for each $n=1,2, \cdots, I_{n} \supseteq I_{n+1}, \alpha\left(S-f^{-1}\left(I_{n}\right)\right)=0$, and $I_{n}$ is of length $1 /\left(2^{n}\right)$. Let $\lambda$ be the unique real number contained in all of the $I_{n}$ 's. Then it is clear that $f(s)=\lambda$ except on a set of $\alpha$ measure zero. In other words for each $f$ in $\mathfrak{F}$ there is a unique real number $P(f)$ such that $f(s)=P(f)$ for "almost all" $s$. It is obvious that $P$ is a non-negative linear functional on $\mathfrak{F}$ which is not identically zero and which does not vanish on characteristic functions of points. It follows then that $P$ is a bounded linear functional on $\mathfrak{F}$ which is not a finite linear combination of point functionals. Conversely suppose that there exists a bounded linear functional on $\mathfrak{F}$ which is not a finite linear combination of point functionals. As we have already remarked we may suppose that this functional is non-negative. Hence by Lemma 2 there exists a non-negative linear functional $P$ on $\mathfrak{F}$ which is not identically zero and which vanishes on characteristic functions of points. For each subset $E$ of $S$ let $\beta(E)=P\left(\phi_{E}\right)$ where $\phi_{E}$ is the characteristic function of $E$. It follows from Lemma 4 that $\beta$ is a countably additive measure function and from Lemma 3 that $\beta$ is not identically zero. Furthermore it is an easy consequence of Lemma 1 that $S=S_{1} \cup S_{2} \cup \cdots \cup S_{n}$ where each $S_{i}$ has the property that for each subset $E$ of $S$ either $\beta\left(E \cap S_{i}\right)=0$ or 
$\beta\left(E \cap S_{i}\right)=\beta\left(S_{i}\right)$. For at least one $i_{0}=1,2, \cdots, n, \beta\left(S_{i_{0}}\right) \neq 0$. Let $\alpha(E)=\beta\left(E \cap S_{i_{0}}\right) / \beta\left(S_{i_{0}}\right)$ for each subset $E$ of $S$. Then it is easily verified that $\alpha$ is a measure function with all properties listed in the statement of the theorem.

Using Ulam's results on two-valued measures we deduce the following corollary.

Corollary. Let $C_{0}=\boldsymbol{\aleph}_{0}$ and for each $n=1,2, \cdots$ let $C_{n}=2^{c_{n-1}}$. Let $D=C_{0}+C_{1}+\cdots$. Then whenever the cardinal of $S$ is less than $D$ every bounded linear functional on $\mathfrak{F}$ is a finite linear combination of point functionals.

\section{REFERENCES}

1. G. Birkhoff, Lattice Theory, Amer. Math. Soc. Colloquium Publications, vol.25, New York, 1940.

2. S. Ulam, Zur Masstheorie in der allgemeinen Mengenlehre, Fund. Math. vol. 16 (1930) pp. 140-150.

HARVARD UNIVERSITY 